\title{
Conformational Changes of Dynamin-Lipid Tubes upon GTP Addition: A Time-Resolved Study Using Digital-Imaging Cryo-TEM
}

\author{
Dganit Danino and Jenny E. Hinshaw
}

Laboratory of Cell Biochemistry and Biology, 8 Center Dr. MSC 0851, NIDDK, NIH, Bethesda, MD 20892

Dynamin is a large GTPase essential for several intracellular budding processes including synaptic vesicle recycling, receptor-mediated endocytosis, caveolae internalization and trafficking from late endosomes and Golgi. During these processes, dynamin is believed to assemble at the necks of budding pits and assist in pinching vesicles from the membrane upon GTP binding and hydrolysis. Using an in vitro assay we have previously shown that dynamin-lipid tubes constrict and vesiculate upon addition of GTP (Sweitzer and Hinshaw; 1998; Zhang and Hinshaw, 2001). In this present study, we are continuing to explore the mechanochemical properties of dynamin by using a time-resolved cryotransmission electron microscopy method.

Wild type recombinant dynamin 1 and a dynamin mutant missing its C-terminal proline rich domain $(\triangle \mathrm{PRD})$ bind to anionic liposomes and form long helical tubular structures, which undergo significant conformational changes upon GTP addition. The stimulated GTPase activity of dynamin is Kcat $\sim 1 \mathrm{sec}^{-1}$, and therefore requires a rapid "fixing" mechanism to reveal the formation of transient intermediates following GTP addition. To fix the specimen, samples were frozen in liquid ethane and examined by cryo-TEM at -180 degrees Celsius. Images were recorded on a side-mount CCD camera, which provides real-time feedback on specimen and image quality, and allows excellent sampling in comparison with imaging on plate [Danino et al., 2001]. Overall, this method is excellent for time-resolved studies of kinetic processes. 

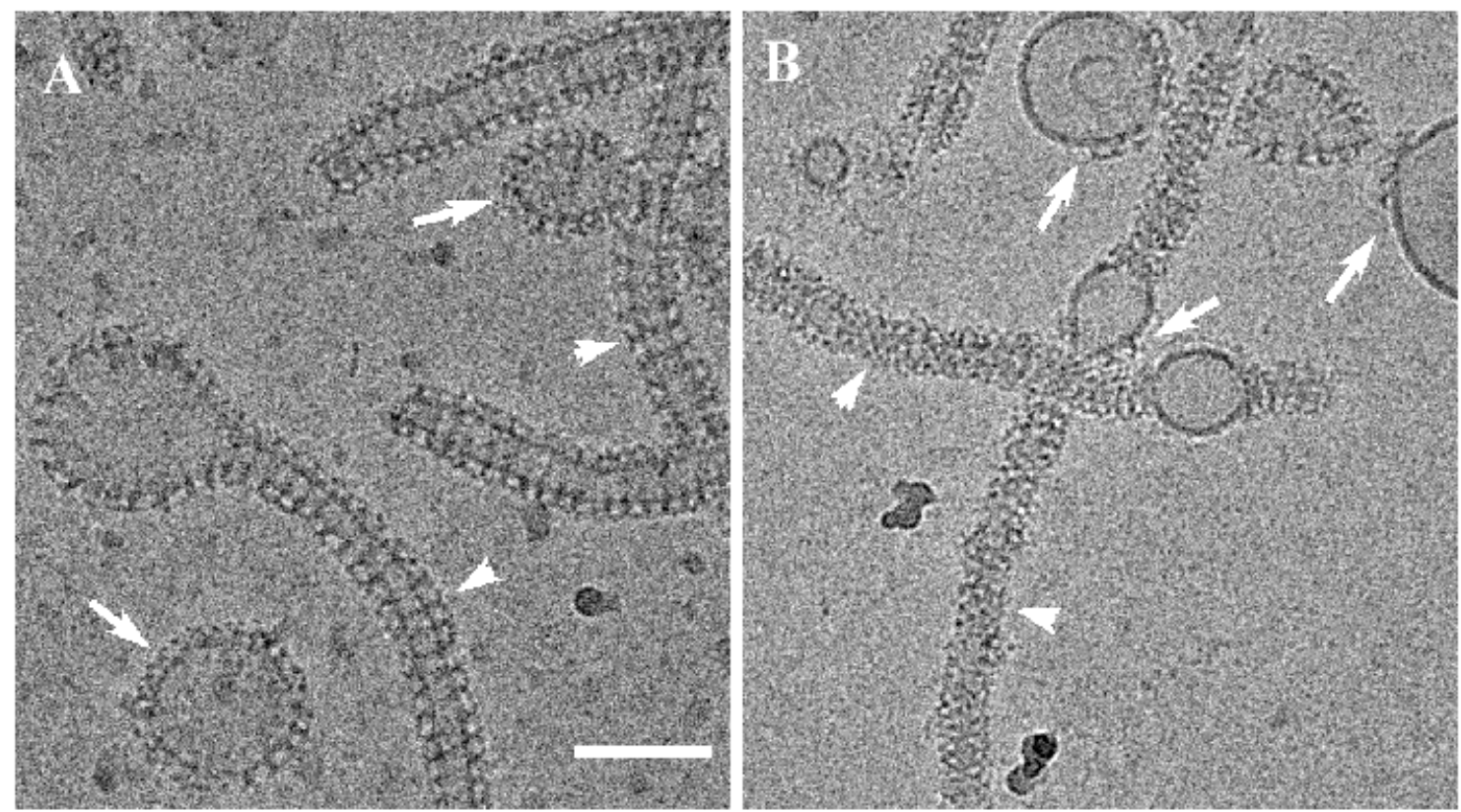

Cryo-TEM images of $\triangle \mathrm{PRD}$ dynamin-lipid structures.

(A) A typical image of dynamin-lipid tubes at time zero (before GTP treatment). The lipid is entirely decorated with dynamin. The T-shape of the dynamin structure (arrowheads), the lipid membrane and the helical striations are clearly seen. (B) 5 seconds after addition of $1 \mathrm{mM}$ GTP constricted tubes with a smaller diameter and shorter pitch are observed (arrowheads). Partially decorated and undecorated lipid domains are also seen at this time point (arrows). Bar $=100 \mathrm{~nm}$.

\section{References:}

[1] Sweitzer SM and Hinshaw JE, Dynamin undergoes a GTP-dependent conformational change causing vesiculation, Cell 1998, 93 (1998)1021.

[2]Zhang P and Hinshaw JE, Three-dimensional reconstruction of dynamin in the constricted state, Nat Cell Biol 3 (2001) 922.

[3] Danino D et al., Digital Cryogenic Transmission Electron Microscopy: an Advanced Tool for Direct Imaging of Complex Fluids, Colloids and Surfaces A: Physicochemical and Engineering Aspects 183 (2001) 113. 